\title{
On coupled fixed point results in asymmetric G-metric spaces
}

\author{
Ravi P Agarwal', Zoran Kadelburg ${ }^{2 *}$ and Stojan Radenović ${ }^{3}$
}

\author{
"Correspondence: \\ kadelbur@matf.bg.ac.rs \\ ${ }^{2}$ Faculty of Mathematics, University \\ of Belgrade, Studentski trg 16, \\ Beograd, 11000, Serbia \\ Full list of author information is \\ available at the end of the article
}

\begin{abstract}
Using a combination of techniques introduced by Jleli and Samet (Fixed Point Theory Appl. 2012:210, 2012) and Samet et al. (Int. J. Anal. 2013:917158, 2013) on the one hand, and by Kadelburg et al. (Bull. Math. Anal. Appl. 4:51-63, 2012) on the other hand, we show that several coupled fixed point results in (ordered) G-metric spaces obtained recently are simple consequences of the respective standard (ordered) metric results. The technique can be applied both in symmetric and asymmetric cases. Moreover, we show by an example that the results thus obtained are usually stronger than those presented in the literature.

MSC: $47 \mathrm{H} 10 ; 54 \mathrm{H} 25$
\end{abstract}

Keywords: G-metric space; coupled fixed point

\section{Introduction}

As one of fruitful generalizations of metric spaces, G-metric spaces were introduced by Mustafa and Sims in [1]. In several subsequent papers, these and other authors obtained many fixed point and common fixed point results, thus extending the known theory from the standard metric case. It should be noted that there exist two kinds of G-metric spaces, symmetric and asymmetric ones, and while it was immediately clear that in the symmetric case these results can be easily reduced to their metric counterparts, in the asymmetric case, new proofs usually had to be found.

The notion of a coupled fixed point for mappings with two variables was introduced in the articles [2-4]. After that, a great number of mathematicians worked in this field and obtained a lot of results in metric and various abstract metric spaces (see, e.g., [5-10]). Coupled fixed points in G-metric spaces were investigated, e.g., in the papers [11-23].

Very recently, some new methods were presented for obtaining fixed point and coupled fixed point results. On the one hand, Jleli and Samet [24] and Samet et al. [25] showed that there is a very simple technique for reducing fixed point results in G-metric spaces, both in symmetric and in asymmetric cases, to their metric counterparts, avoiding complicated proofs from the known papers. On the other hand, in the papers [26-33], the authors presented another technique which reduces coupled fixed point results in metric and various abstract metric spaces to the results for mappings with one variable. This technique was used by Kadelburg et al. [34] and afterwards by Agarwal and Karapinar [35] to obtain coupled fixed point results in symmetric $G$-metric spaces.

By combining the mentioned techniques, we show in this paper that several coupled fixed point results in (ordered) G-metric spaces obtained in recent years and presented in

\section{Springer}

C2013 Agarwal et al.: licensee Springer. This is an Open Access article distributed under the terms of the Creative Commons Attribution License (http://creativecommons.org/licenses/by/2.0), which permits unrestricted use, distribution, and reproduction in any medium, provided the original work is properly cited. 
the papers [11-23] simply follow from the well-known standard (ordered) metric results for mappings with one variable. The technique can be applied in both symmetric and asymmetric cases. Moreover, we will show by an example that the results obtained in this way are usually stronger and can be applied in a greater number of cases.

\section{Preliminaries}

For more details on the following definitions and results concerning G-metric spaces, we refer the reader to [1].

Definition 1 Let $\mathcal{X}$ be a nonempty set, and let $g: \mathcal{X}^{3} \rightarrow \mathbb{R}^{+}$be a function satisfying the following properties:

(G1) $g(x, y, z)=0$ if $x=y=z$;

(G2) $0<g(x, x, y)$ for all $x, y \in \mathcal{X}$ with $x \neq y$;

(G3) $g(x, x, y) \leq g(x, y, z)$ for all $x, y, z \in \mathcal{X}$ with $z \neq y$;

(G4) $g(x, y, z)=g(x, z, y)=g(y, z, x)=\cdots$ (symmetry in all three variables);

(G5) $g(x, y, z) \leq g(x, a, a)+g(a, y, z)$ for all $x, y, z, a \in \mathcal{X}$ (rectangle inequality).

Then the function $g$ is called a $G$-metric on $\mathcal{X}$ and the pair $(\mathcal{X}, g)$ is called a $G$-metric space.

Definition 2 Let $(\mathcal{X}, g)$ be a $G$-metric space, and let $\left\{x_{n}\right\}$ be a sequence of points in $\mathcal{X}$.

1. A point $x \in \mathcal{X}$ is said to be the limit of a sequence $\left\{x_{n}\right\}$ if $\lim _{n, m \rightarrow \infty} g\left(x, x_{n}, x_{m}\right)=0$, and one says that the sequence $\left\{x_{n}\right\}$ is $g$-convergent to $x$.

2. The sequence $\left\{x_{n}\right\}$ is said to be a $G$-Cauchy sequence if, for every $\varepsilon>0$, there is a positive integer $N$ such that $g\left(x_{n}, x_{m}, x_{l}\right)<\varepsilon$ for all $n, m, l \geq N$; that is, if $g\left(x_{n}, x_{m}, x_{l}\right) \rightarrow 0$ as $n, m, l \rightarrow \infty$.

3. $(\mathcal{X}, g)$ is said to be $G$-complete (or a complete $G$-metric space) if every $G$-Cauchy sequence in $(\mathcal{X}, g)$ is $G$-convergent in $\mathcal{X}$.

It was shown in [1] that a G-metric induces a Hausdorff topology and that the convergence, as described in the above definition, is relative to this topology. The topology being Hausdorff, a sequence can converge to at most one point.

Definition 3 A $G$-metric space $(\mathcal{X}, g)$ is called symmetric if

$$
g(x, y, y)=g(x, x, y)
$$

holds for all $x, y \in \mathcal{X}$.

The following are some simple examples of $G$-metric spaces.

Example $1(1)$ Let $(\mathcal{X}, d)$ be an ordinary metric space. Define $g$ by

$$
g(x, y, z)=d(x, y)+d(y, z)+d(x, z)
$$

for all $x, y, z \in \mathcal{X}$. Then it is clear that $(\mathcal{X}, g)$ is a symmetric $G$-metric space.

(2) Let $\mathcal{X}=\{a, b\}$. Define

$$
g(a, a, a)=g(b, b, b)=0, \quad g(a, a, b)=1, \quad g(a, b, b)=2,
$$


and extend $g$ to $\mathcal{X}^{3}$ by using the symmetry in the variables. Then it is clear that $(\mathcal{X}, g)$ is an asymmetric $G$-metric space.

Remark 1 If $(\mathcal{X}, g)$ is a $G$-metric space, then

$$
d_{g}(x, y)=g(x, x, y)+g(x, y, y)
$$

defines a standard metric on $\mathcal{X}$. If the $G$-metric $g$ is symmetric, this reduces to $d_{g}(x, y)=$ $2 g(x, x, y)$. This simple fact implies that most of the fixed point results in symmetric $G$-metric spaces can be easily reduced to their metric counterparts. In the asymmetric case, another approach is needed.

Definition $4[3,4,36]$ Let $(\mathcal{X}, \preceq)$ be a partially ordered set, $f: \mathcal{X}^{2} \rightarrow \mathcal{X}$ and $h: \mathcal{X} \rightarrow \mathcal{X}$.

1. $f$ is said to have the $h$-mixed monotone property if the following two conditions are satisfied:

$$
\begin{array}{ll}
\left(\forall x_{1}, x_{2}, y \in \mathcal{X}\right) \quad h x_{1} \preceq h x_{2} \quad \Longrightarrow \quad f\left(x_{1}, y\right) \preceq f\left(x_{2}, y\right), \\
\left(\forall x, y_{1}, y_{2} \in \mathcal{X}\right) \quad h y_{1} \preceq h y_{2} \quad \Longrightarrow \quad f\left(x, y_{1}\right) \succeq f\left(x, y_{2}\right) .
\end{array}
$$

If $h=i_{\mathcal{X}}$ (the identity map), we say that $f$ has the mixed monotone property.

2. A point $(x, y) \in \mathcal{X} \times \mathcal{X}$ is said to be a coupled coincidence point of $f$ and $h$ if $f(x, y)=h x$ and $f(y, x)=h y$, and their common coupled fixed point if $f(x, y)=h x=x$ and $f(y, x)=h y=y$.

3. The mappings $f$ and $h$ are called $w$-compatible if $f(h x, h y)=h(f(x, y))$ and $f(h y, h x)=h(f(y, x))$ whenever $f(x, y)=h x$ and $f(y, x)=h y$.

If $\mathcal{X}$ is a nonempty set, then the triple $(\mathcal{X}, g, \preceq)$ is called an ordered $G$-metric space if:

(i) $(\mathcal{X}, g)$ is a $G$-metric space, and

(ii) $(\mathcal{X}, \preceq)$ is a partially ordered set.

\section{Main results}

We will use the following simple lemma for obtaining our results.

Lemma 1 Let $(\mathcal{X}, g, \preceq)$ be an ordered G-metric space.

(a) If a relation $\sqsubseteq$ is defined on $\mathcal{X}^{2}$ by

$$
X \sqsubseteq U \quad \Longleftrightarrow \quad x \preceq u \wedge y \succeq v, \quad X=(x, y), U=(u, v) \in \mathcal{X}^{2},
$$

and mappings $D_{g}: \mathcal{X}^{2} \times \mathcal{X}^{2} \rightarrow \mathbb{R}^{+}$and $\Delta_{g}: \mathcal{X}^{2} \times \mathcal{X}^{2} \rightarrow \mathbb{R}^{+}$are given by

$$
\begin{aligned}
& D_{g}(X, U)=g(x, x, u)+g(x, u, u)+g(y, y, v)+g(y, v, v), \\
& \Delta_{g}(X, U)=\max \{g(x, x, u), g(x, u, u), g(y, y, v), g(y, v, v)\}
\end{aligned}
$$

for $X=(x, y), U=(u, v) \in \mathcal{X}^{2}$, then $\left(\mathcal{X}^{2}, D_{g}, \sqsubseteq\right)$ and $\left(\mathcal{X}^{2}, \Delta_{g}, \sqsubseteq\right)$ are ordered $G$-metric spaces. The spaces $\left(\mathcal{X}^{2}, D_{g}\right)$ and $\left(\mathcal{X}^{2}, \Delta_{g}\right)$ are complete iff $(\mathcal{X}, g)$ is complete. 
(b) If $h: \mathcal{X} \rightarrow \mathcal{X}$ is a self-map, and a mapping $f: \mathcal{X}^{2} \rightarrow \mathcal{X}$ has the h-mixed monotone property, then the mapping $F: \mathcal{X}^{2} \rightarrow \mathcal{X}^{2}$ given by

$$
F X=(f(x, y), f(y, x)), \quad X=(x, y) \in \mathcal{X}^{2}
$$

is $H$-nondecreasing w.r.t. $\sqsubseteq$, i.e.,

$$
H X \sqsubseteq H U \quad \Longrightarrow \quad F X \sqsubseteq F U
$$

where $H: \mathcal{X}^{2} \rightarrow \mathcal{X}^{2}$ is defined by $H(x, y)=(h x, h y)$.

(c) If $h$ is continuous in $(\mathcal{X}, g)$, then $H$ is continuous in both $\left(\mathcal{X}^{2}, D_{g}\right)$ and $\left(\mathcal{X}^{2}, \Delta_{g}\right)$. Iff is continuous from $\left(\mathcal{X}^{2}, D_{g}\right)$ to $(\mathcal{X}, g)$ (resp. from $\left(\mathcal{X}^{2}, \Delta_{g}\right)$ to $(\mathcal{X}, g)$ ), then $F$ is continuous in $\left(\mathcal{X}^{2}, D_{g}\right)$ (resp.in $\left.\left(\mathcal{X}^{2}, \Delta_{g}\right)\right)$.

Proof We will only check the second part of assertion (a); the proofs of all other parts are straightforward.

It was stated already in [1] that if $g$ is a $G$-metric on $\mathcal{X}$, then

$$
\begin{aligned}
& d_{g}(x, y)=g(x, x, y)+g(x, y, y), \\
& \delta_{g}(x, y)=\max \{g(x, x, y), g(x, y, y)\}
\end{aligned}
$$

define standard metrics on $\mathcal{X}$, and that topologies thus generated are the same as the topology of $(\mathcal{X}, g)$. In particular, the completeness is satisfied simultaneously.

On the other hand, it is well known that for each (standard) metric space $(\mathcal{X}, d)$, the mappings

$$
\begin{aligned}
& D(X, U)=d(x, u)+d(y, v), \\
& \Delta(U, V)=\max \{d(x, u), d(y, v)\}
\end{aligned}
$$

for $X=(x, y), U=(u, v) \in \mathcal{X}^{2}$ are metrics on $\mathcal{X}^{2}$, also preserving the completeness property. Combining these two facts, we obtain that the mappings

$$
\begin{aligned}
D_{g}(X, U) & =d_{g}(x, u)+d_{g}(y, v) \\
& =g(x, x, u)+g(x, u, u)+g(y, y, v)+g(y, v, v), \\
\Delta_{g}(X, U) & =\max \left\{d_{g}(x, u), d_{g}(y, v)\right\} \\
& =\max \{g(x, x, u), g(x, u, u), g(y, y, v), g(y, v, v)\}
\end{aligned}
$$

satisfy all the stated properties.

Remark 2 If the given $G$-metric space $(\mathcal{X}, g)$ is symmetric, then, using the construction given in [34, Lemma 3.1] and afterwards in [35, Sections 4 and 5], we can obtain the previous result in a slightly different way. Namely, in this case, one can consider the G-metric $G$ on $\mathcal{X}^{2}$ given by

$$
G(X, U, S)=g(x, u, s)+g(y, v, t), \quad X=(x, y), U=(u, v), S=(s, t) \in \mathcal{X}^{2},
$$


and then associate the metrics

$$
\begin{aligned}
& d_{G}(X, U)=G(X, X, U)+G(X, U, U), \\
& \delta_{G}(X, U)=\max \{G(X, X, U), G(X, U, U)\} .
\end{aligned}
$$

It is easy to see that, in fact, $d_{G} \equiv D_{g}$ and $\delta_{G} \equiv \Delta_{g}$.

However, this approach cannot be used in the asymmetric case, since in this case, (3.1) does not define a G-metric on $\mathcal{X}^{2}$ (see [1, Section 4] and, further, Example 2).

Now we are ready to state some of our main results. We start, as a sample, with the following theorem.

Theorem 1 Let $(\mathcal{X}, g, \preceq)$ be a complete partially ordered G-metric space, and let $f: \mathcal{X}^{2} \rightarrow$ $\mathcal{X}$ and $h: \mathcal{X} \rightarrow \mathcal{X}$ be mappings such that $f\left(\mathcal{X}^{2}\right) \subseteq h(\mathcal{X}), h(\mathcal{X})$ is closed and $f$ has the mixed $h$-monotone property. Suppose that there exist $x_{0}, y_{0} \in \mathcal{X}$ such that $h x_{0} \preceq f\left(x_{0}, y_{0}\right)$ and $h y_{0} \succeq f\left(y_{0}, x_{0}\right)$. Suppose also that there exists $\lambda \in[0,1)$ such that

$$
\begin{aligned}
& g(f(x, y), f(x, y), f(s, t))+g(f(x, y), f(s, t), f(s, t)) \\
& \quad+g(f(y, x), f(y, x), f(t, s))+g(f(y, x), f(t, s), f(t, s)) \\
& \leq \lambda[g(h x, h x, h s)+g(h x, h s, h s)+g(h y, h y, h t)+g(h y, h t, h t)]
\end{aligned}
$$

for all $x, y, s, t \in \mathcal{X}$ satisfying ( $h x \preceq h s$ and $h y \succeq h t)$ or ( $h x \succeq h s$ and $h y \preceq h t)$. Let us assume also that if $\left\{h x_{n}\right\}$ is a nondecreasing sequence in $\mathcal{X}$ converging to some $h z \in h(\mathcal{X})$, then $h x_{n} \preceq h z \preceq h(h z)$ for each $n \in \mathbb{N}$. Then the mappings $f$ and $h$ have a coupled coincidence point. Moreover, if $f$ and $h$ are w-compatible, then $f$ and $h$ have a common coupled fixed point.

Proof Consider the complete partially ordered metric space $\left(\mathcal{X}^{2}, D_{g}\right.$, 드) and the mappings $F: \mathcal{X}^{2} \rightarrow \mathcal{X}^{2}$ and $H: \mathcal{X}^{2} \rightarrow \mathcal{X}^{2}$ defined in Lemma 1 . Then, obviously, the following conditions hold:

1. $F\left(\mathcal{X}^{2}\right) \subseteq H\left(\mathcal{X}^{2}\right)$ and $H\left(\mathcal{X}^{2}\right)$ is closed;

2. $F$ is $H$-nondecreasing;

3. There exists $X_{0} \in \mathcal{X}^{2}$ such that $H X_{0} \sqsubseteq F X_{0}$.

Moreover, it follows from (3.2) that there exists $\lambda \in[0,1)$ such that

$$
D_{g}(F X, F S) \leq \lambda D_{g}(H X, H S)
$$

holds for all $X, S \in \mathcal{X}^{2}$ such that $H X \sqsubseteq H S$ or $H X \sqsupseteq H S$. Now, all the conditions of a special case of [37, Theorem 2.2] are fulfilled and it follows that the mappings $F$ and $H$ have a coincidence point $X=(x, y) \in \mathcal{X}^{2}$ which is, obviously, a coupled coincidence point of $f$ and $h$. The last assertion also follows easily.

Corollary 1 Let $(\mathcal{X}, g, \preceq)$ be a complete partially ordered G-metric space, and let $f: \mathcal{X}^{2} \rightarrow$ $\mathcal{X}$ and $h: \mathcal{X} \rightarrow \mathcal{X}$ be mappings such that $f\left(\mathcal{X}^{2}\right) \subseteq h(\mathcal{X}), h(\mathcal{X})$ is closed and $f$ has the 
mixed $h$-monotone property. Suppose that there exist $x_{0}, y_{0} \in \mathcal{X}$ such that $h x_{0} \preceq f\left(x_{0}, y_{0}\right)$ and $h y_{0} \succeq f\left(y_{0}, x_{0}\right)$. Suppose also that there exists $\lambda \in[0,1)$ such that

$$
\begin{aligned}
& g(f(x, y), f(u, v), f(s, t))+g(f(y, x), f(v, u), f(t, s)) \\
& \quad \leq \lambda[g(h x, h u, h s)+g(h y, h v, h t)]
\end{aligned}
$$

for all $x, y, u, v, s, t \in \mathcal{X}$ satisfying ( $h x \preceq h u \preceq h s$ and $h y \succeq h v \succeq h t)$ or $(h x \succeq h u \succeq h s$ and $h y \preceq h v \preceq h t)$. Let us assume also that if $\left\{h x_{n}\right\}$ is a nondecreasing sequence in $\mathcal{X}$ converging to some $h z \in h(\mathcal{X})$, then $h x_{n} \preceq h z \preceq h(h z)$ for each $n \in \mathbb{N}$. Then the mappings $f$ and $h$ have a coupled coincidence point. Moreover, if $f$ and $h$ are $w$-compatible, then $f$ and $h$ have a common coupled fixed point.

Proof We have only to prove that condition (3.4) implies condition (3.2). Indeed, putting $u=x$ and $v=y$ in (3.4), we get that

$$
\begin{aligned}
& g(f(x, y), f(x, y), f(s, t))+g(f(y, x), f(y, x), f(t, s)) \\
& \quad \leq \lambda[g(h x, h x, h s)+g(h y, h y, h t)],
\end{aligned}
$$

and putting $u=s$ and $v=t$, we get

$$
\begin{aligned}
& g(f(x, y), f(s, t), f(s, t))+g(f(y, x), f(t, s), f(t, s)) \\
& \quad \leq \lambda[g(h x, h s, h s)+g(h y, h t, h t)] .
\end{aligned}
$$

Adding up, and taking into account the definitions of mappings $F$ and $H$ as well as the definition of metric $D_{g}$, we obtain condition (3.2) (i.e., condition (3.3)).

In an even easier way, one can obtain the following versions of the previous results in the space without order. In this case, the given technique reduces the problem simply to the Banach contraction principle.

Theorem 2 Let $(\mathcal{X}, g)$ be a complete G-metric space, and let $f: \mathcal{X}^{2} \rightarrow \mathcal{X}$ and $h: \mathcal{X} \rightarrow \mathcal{X}$ be mappings such that $f\left(\mathcal{X}^{2}\right) \subseteq h(\mathcal{X})$ and $h(\mathcal{X})$ is closed. Suppose also that there exists $\lambda \in[0,1)$ such that condition (3.2) holds for all $x, y, s, t \in \mathcal{X}$. If $h$ is continuous, then the mappings $f$ and $h$ have a coupled coincidence point. Moreover, iff and h are w-compatible, then $f$ and h have a common coupled fixed point.

Corollary 2 Let $(\mathcal{X}, g)$ be a complete G-metric space, and let $f: \mathcal{X}^{2} \rightarrow \mathcal{X}$ and $h: \mathcal{X} \rightarrow \mathcal{X}$ be mappings such that $f\left(\mathcal{X}^{2}\right) \subseteq h(\mathcal{X})$ and $h(\mathcal{X})$ is closed. Suppose also that there exists $\lambda \in[0,1)$ such that condition (3.4) holds for all $x, y, u, v, s, t \in \mathcal{X}$. If $h$ is continuous, then the mappings $f$ and $h$ have a coupled coincidence point. Moreover, iff and h are w-compatible, then $f$ and $h$ have a common coupled fixed point.

Remark 3 The last result was obtained (in the special case $h=i_{X}$ ) in [34, Corollary 4.1] and afterwards (implicitly) in the course of proof of [35, Theorem 5.3], but in the case when the given $G$-metric $g$ is symmetric. The proof from these articles cannot be applied in the asymmetric case (see Remark 2 and, further, Example 2). 
Remark 4 The obtained results are strict improvements of some results obtained earlier. In the symmetric case, this was shown by Kadelburg et al. (see [34, Example 4.1]). We present an example in an asymmetric case.

Example 2 Let $(\mathcal{X}, g)$ be the $G$-metric space considered in [38, Example 3.4], i.e., let $\mathcal{X}=$ $\{0,1,2\}$ and $g: \mathcal{X}^{3} \rightarrow \mathbb{R}^{+}$be given as

$$
g(x, y, z)= \begin{cases}0, & \text { if } x=y=z \\ 1, & \text { if }(x, y, z) \in\{(0,0,1),(0,1,1),(0,2,2),(1,2,2)\} \\ 2, & \text { if }(x, y, z) \in\{(0,0,2),(1,1,2),(0,1,2)\}\end{cases}
$$

and extended by symmetry in the variables. Then it is easy to check that $g$ is a $G$-metric which is asymmetric since $g(0,0,2) \neq g(0,2,2)$ and $g(1,1,2) \neq g(1,2,2)$.

Let $h: \mathcal{X} \rightarrow \mathcal{X}$ be given as $h x=x$, and let $f: \mathcal{X}^{2} \rightarrow \mathcal{X}$ be defined by

$$
f:\left(\begin{array}{ccccccccc}
(0,0) & (0,1) & (0,2) & (1,0) & (1,1) & (1,2) & (2,0) & (2,1) & (2,2) \\
0 & 0 & 0 & 0 & 0 & 0 & 1 & 1 & 1
\end{array}\right) .
$$

We will show first that $f$ and $h$ satisfy neither the condition

$$
g(f(x, y), f(u, v), f(s, t)) \leq \frac{\lambda}{2}[g(h x, h u, h s)+g(h y, h v, h t)]
$$

for any $\lambda \in[0,1)$ (which is condition (3.1) from [15]) nor (weaker) condition (3.4). Indeed, take, e.g., $x=u=s=y=1$ and $v=t=2$ and condition (3.4) becomes

$$
\begin{aligned}
& g(f(1,1), f(1,2), f(1,2))+g(f(1,1), f(2,1), f(2,1)) \\
& \quad=g(0,0,0)+g(0,1,1)=1 \leq \lambda=\lambda[g(1,1,1)+g(1,2,2)],
\end{aligned}
$$

which is a contradiction for any $\lambda \in[0,1)$. Hence, neither $[15$, Theorem 3.1] nor Corollary 2 can be used to conclude that $f$ and $h$ have a coupled coincidence point (i.e., that $f$ has a coupled fixed point).

In order to show that $f$ and $h$ satisfy the conditions of Theorem 2, we first note that $d_{g}(0,1)=2, d_{g}(0,2)=d_{g}(1,2)=3$ and

$$
\begin{aligned}
& D_{g}((0,0),(0,1))=D_{g}((0,0),(1,0))=D_{g}((0,1),(1,1))=D_{g}((0,2),(1,2)) \\
& =D_{g}((1,0),(1,1))=D_{g}((2,0),(2,1))=2, \\
& \begin{aligned}
D_{g}((0,0),(0,2)) & =D_{g}((0,0),(2,0))=D_{g}((0,1),(0,2))=D_{g}((0,1),(2,1)) \\
& =D_{g}((0,2),(2,2))=D_{g}((1,0),(1,2))=D_{g}((1,0),(2,0)) \\
& =D_{g}((1,1),(1,2))=D_{g}((1,1),(2,1))=D_{g}((1,2),(2,2)) \\
& =D_{g}((2,0),(2,2))=D_{g}((2,1),(2,2))=3, \\
D_{g}((0,0),(1,1)) & =D_{g}((0,1),(1,0))=4, \\
D_{g}((0,0),(1,2)) & =D_{g}((0,0),(2,1))=D_{g}((0,1),(1,2))=D_{g}((0,1),(2,0))
\end{aligned}
\end{aligned}
$$




$$
\begin{aligned}
& =D_{g}((0,2),(1,0))=D_{g}((0,2),(1,1))=D_{g}((1,0),(2,1)) \\
& =D_{g}((1,1),(2,0))=5, \\
D_{g}((0,0),(2,2)) & =D_{g}((0,1),(2,2))=D_{g}((0,2),(2,0))=D_{g}((0,2),(2,1)) \\
& =D_{g}((1,0),(2,2))=D_{g}((1,1),(2,2))=D_{g}((1,2),(2,0)) \\
& =D_{g}((1,2),(2,1))=6 .
\end{aligned}
$$

Take now $\lambda=\frac{2}{3}$. By a careful calculation (there are 21 nontrivial cases) it can be checked that condition (3.2) (i.e., condition (3.3)) is satisfied for all $X, S \in \mathcal{X}^{2}$.

Hence, Theorem 2 can be applied to conclude that $f$ has a coupled fixed point (which is $(0,0))$.

We note also that the approach from the papers $[34,35]$ cannot be used in this example since

$$
G((x, y),(u, v),(s, t))=g(x, u, s)+g(y, v, t)
$$

does not define a $G$-metric on $\mathcal{X}^{2}$. Indeed, e.g.,

$$
\begin{aligned}
G((0,2),(0,2),(2,0)) & =g(0,0,2)+g(2,2,0)=2+1=3 \\
& >2=1+1=g(0,2,2)+g(2,0,2)=G((0,2),(2,0),(2,2)),
\end{aligned}
$$

although $(2,0) \neq(2,2)$, and the property (G3) of $G$-metrics is not satisfied.

For the sake of simplicity, we shall consider in the rest of the paper 'unordered' versions of coupled fixed point results. 'Ordered' versions can be formulated and proved using usual variations.

The proof of our next result uses the metric $\Delta_{g}$.

Theorem 3 Let $(\mathcal{X}, g)$ be a complete G-metric space, and let $f: \mathcal{X}^{2} \rightarrow \mathcal{X}$. Suppose that there exists $\lambda \in[0,1)$ such that for all $x, u, s, y, v, t \in \mathcal{X}$ the following inequality holds:

$$
\begin{aligned}
& g(f(x, y), f(u, v), f(s, t)) \\
& \quad \leq \lambda \max \{g(x, u, s), g(x, f(x, y), f(x, y)), g(u, f(u, v), f(u, v)), g(s, f(s, t), f(s, t))\} .
\end{aligned}
$$

Then $f$ has a unique coupled fixed point in $\mathcal{X}^{2}$ and it is of the form $(x, x)$ for some $x \in \mathcal{X}$.

Proof Consider the complete metric space $\left(\mathcal{X}^{2}, \Delta_{g}\right)$ and the mapping $F: \mathcal{X}^{2} \rightarrow \mathcal{X}^{2}$ defined in Lemma 1. Putting $u=x$ and $v=y$ in (3.5), one gets

$$
g(f(x, y), f(x, y), f(s, t)) \leq \lambda \max \{g(x, x, s), g(x, f(x, y), f(x, y)), g(s, f(s, t), f(s, t))\} ;
$$

interchanging the places of $x$ and $y$, as well as of $s$ and $t$, this gives

$$
g(f(y, x), f(y, x), f(t, s)) \leq \lambda \max \{g(y, y, t), g(y, f(y, x), f(y, x)), g(t, f(t, s), f(t, s))\} .
$$


Putting now $u=s$ and $v=t$ in (3.5), one gets

$$
g(f(x, y), f(s, t), f(s, t)) \leq \lambda \max \{g(x, s, s), g(x, f(x, y), f(x, y)), g(s, f(s, t), f(s, t))\}
$$

interchanging the places of $x$ and $y$, as well as of $s$ and $t$, this gives

$$
g(f(y, x), f(t, s), f(t, s)) \leq \lambda \max \{g(y, t, t), g(y, f(y, x), f(y, x)), g(t, f(t, s), f(t, s))\}
$$

Taking into account the definition of metric $\Delta_{g}$ in Lemma 1, it follows from the last four inequalities that

$$
\Delta_{g}(F X, F S) \leq \lambda \max \left\{\Delta_{g}(X, S), \Delta_{g}(X, F X), \Delta_{g}(S, F S)\right\}
$$

holds for all $X, S \in \mathcal{X}^{2}$. By a well-known result from the theory of standard metric spaces (see, e.g., [39]), it follows that there exists a unique point $X=(x, y) \in \mathcal{X}^{2}$ such that $F X=X$. Obviously, $(x, y)$ is a coupled fixed point of the mapping $f$. Since the coupled fixed point is unique, it must be of the form $(x, x)$ for some $x \in \mathcal{X}$.

When considering situations where contractive conditions are of 'weak' kind, or they use the so-called comparison functions, a variation of the previous approach is needed. One possibility is to use quasi-metrics. Recall that a pair $(\mathcal{X}, d)$ is called a quasi-metric space if the mapping $d$ has all the properties of a metric except, possibly, the symmetry $d(x, y)=d(y, x)$. For some properties of quasi-metric spaces, we refer to [24]. In particular, the following fixed point result was proved in that paper.

Theorem 4 [24, Theorem 3.2] Let $(\mathcal{X}, d)$ be a complete quasi-metric space, and let $T$ : $\mathcal{X} \rightarrow \mathcal{X}$ be a mapping satisfying

$$
d(T x, T y) \leq d(x, y)-\varphi(d(x, y))
$$

for all $x, y \in X$, where $\varphi:[0,+\infty) \rightarrow[0,+\infty)$ is continuous with $\varphi^{-1}(\{0\})=\{0\}$. Then $T$ has a unique fixed point.

Remark 5 An additional nondecreasing function $\psi$ could be added in (3.6) to become

$$
\psi(d(T x, T y)) \leq \psi(d(x, y))-\varphi(d(x, y))
$$

However, it was shown in [40] that it is redundant, hence we will not use it here.

Obviously, if $(\mathcal{X}, g)$ is a $G$-metric space, then

$$
d_{g}^{\prime}(x, y)=g(x, y, y)
$$

defines a quasi-metric on $\mathcal{X}$. Moreover,

$$
D_{g}^{\prime}(X, U)=g(x, u, u)+g(y, v, v), \quad X=(x, y), U=(u, v) \in \mathcal{X}^{2}
$$

defines a quasi-metric on $\mathcal{X}^{2}$. The space $\left(\mathcal{X}^{2}, D_{g}^{\prime}\right)$ is complete iff $(\mathcal{X}, g)$ is $G$-complete. Now we can easily prove the following theorem. 
Theorem 5 Let $(\mathcal{X}, g)$ be a complete G-metric space, and let $f: \mathcal{X}^{2} \rightarrow \mathcal{X}$ satisfy

$$
\begin{array}{r}
g(f(x, y), f(u, v), f(u, v))+g(f(y, x), f(v, u), f(v, u)) \\
\quad \leq g(x, u, u)+g(y, v, v)-\varphi(g(x, u, u)+g(y, v, v))
\end{array}
$$

for all $x, y, u, v \in \mathcal{X}$, where $\varphi:[0,+\infty) \rightarrow[0,+\infty)$ is continuous with $\varphi^{-1}(\{0\})=\{0\}$. Then $f$ has a unique coupled fixed point.

Proof Consider the (complete) quasi-metric space $\left(\mathcal{X}^{2}, D_{g}^{\prime}\right)$ given by (3.7) and the selfmapping $F$ given on $\mathcal{X}^{2}$ by $F X=(f(x, y), f(y, x))$ for $X=(x, y) \in \mathcal{X}^{2}$. Then contractive condition (3.8) gives

$$
D_{g}^{\prime}(F X, F U) \leq D_{g}^{\prime}(X, U)-\varphi\left(D_{g}^{\prime}(X, U)\right)
$$

for $X, U \in \mathcal{X}^{2}$. Hence, Theorem 4 can be applied to conclude that $F$ has a unique fixed point, which is then a coupled fixed point of $f$.

Another possibility is to impose an additional condition on the function $\varphi$, or on the comparison function $\Phi$. This is illustrated in the next result.

Theorem 6 Let $(\mathcal{X}, g)$ be a complete $G$-metric space, and let $f: \mathcal{X}^{2} \rightarrow \mathcal{X}$ satisfy the condition

$$
g(f(x, y), f(u, v), f(s, t)) \leq \Phi\left(\frac{g(x, u, s)+g(y, v, t)}{2}\right)
$$

for all $x, y, u, v, s, t \in \mathcal{X}$, where $\Phi:[0,+\infty) \rightarrow[0,+\infty)$ is right-continuous, $\Phi(t)<t$ for $t>0$ and $\Phi(a+b) \geq \Phi(a)+\Phi(b)$ for $a, b \in[0,+\infty)$. Then $f$ has a unique coupled fixed point.

Proof Consider again the space $\left(\mathcal{X}^{2}, D_{g}\right)$ and the mapping $F$ given in Lemma 1. Putting $u=x$ and $v=y$ in (3.9), we get

$$
g(f(x, y), f(x, y), f(s, t)) \leq \Phi\left(\frac{g(x, x, s)+g(y, y, t)}{2}\right)
$$

interchanging places of $x$ and $y$, as well as $s$ and $t$, we obtain

$$
g(f(y, x), f(y, x), f(t, s)) \leq \Phi\left(\frac{g(x, x, s)+g(y, y, t)}{2}\right) .
$$

On the other hand, putting $u=s$ and $v=t$ in (3.9), we get

$$
g(f(x, y), f(s, t), f(s, t)) \leq \Phi\left(\frac{g(x, s, s)+g(y, t, t)}{2}\right)
$$

interchanging places of $x$ and $y$, as well as $s$ and $t$, we obtain

$$
g(f(y, x), f(t, s), f(t, s)) \leq \Phi\left(\frac{g(x, s, s)+g(y, t, t)}{2}\right) .
$$


Adding up the last four inequalities, and using the assumed properties of function $\Phi$, we get that

$$
\begin{aligned}
D_{g}(F X, F S) \leq & \Phi\left(\frac{g(x, x, s)+g(y, y, t)}{2}\right)+\Phi\left(\frac{g(x, x, s)+g(y, y, t)}{2}\right) \\
& +\Phi\left(\frac{g(x, s, s)+g(y, t, t)}{2}\right)+\Phi\left(\frac{g(x, s, s)+g(y, t, t)}{2}\right) \\
\leq & \Phi(g(x, x, s)+g(y, y, t)+g(x, s, s)+g(y, t, t))=\Phi\left(D_{g}(X, S)\right)
\end{aligned}
$$

for all $X=(x, y), S=(s, t) \in \mathcal{X}^{2}$. Hence, by a classical result of Boyd and Wong [41], it follows that $F$ has a unique fixed point, which is then a coupled fixed point of $f$.

It is clear that a lot of other known coupled fixed point results in G-metric spaces (both symmetric and asymmetric) can be easily obtained in this way.

\section{Competing interests}

The authors declare that they have no competing interests.

\section{Authors' contributions}

All authors contributed equally and significantly in writing this paper. All authors read and approved the final manuscript.

\section{Author details}

'Department of Mathematics, Texas A\&M University-Kingsville, 700 University Blvd., Kingsville, TX 78363-8202, USA. ${ }^{2}$ Faculty of Mathematics, University of Belgrade, Studentski trg 16, Beograd, 11000, Serbia. ${ }^{3}$ Faculty of Mechanical Engineering, University of Belgrade, Kraljice Marije 16, Beograd, 11120, Serbia.

\section{Acknowledgements}

The authors are indebted to the referees whose suggestions helped them to improve the exposition. The second and third authors are thankful to the Ministry of Education, Science and Technological Development of Serbia.

\section{Received: 13 March 2013 Accepted: 2 September 2013 Published: 11 Nov 2013}

\section{References}

1. Mustafa, Z, Sims, B: A new approach to generalized metric spaces. J. Nonlinear Convex Anal. 7(2), $289-297$ (2006)

2. Guo, D, Lakshmikantham, V: Coupled fixed points of nonlinear operators with applications. Nonlinear Anal. 11 , 623-632 (1987)

3. Bhaskar, TG, Lakshmikantham, V: Fixed point theorems in partially ordered metric spaces and applications. Nonlinear Anal. 65, 1379-1393 (2006)

4. Lakshmikantham, V, Ćirić, L: Coupled fixed point theorems for nonlinear contractions in partially ordered metric spaces. Nonlinear Anal. 70, 4341-4349 (2009)

5. Sintunavarat, W, Cho, YJ, Kumam, P: Coupled coincidence point theorems for contractions without commutative condition in intuitionistic fuzzy normed spaces. Fixed Point Theory Appl. 2011, 81 (2011)

6. Sintunavarat, W, Cho, YJ, Kumam, P: Coupled fixed point theorems for weak contraction mapping under F-invariant set. Abstr. Appl. Anal. 2012, Article ID 324874 (2012)

7. Sintunavarat, W, Cho, YJ, Kumam, P: Coupled fixed point theorem for contraction mapping induced by cone ball-metric in partially ordered spaces. Fixed Point Theory Appl. 2012, 128 (2012)

8. Sintunavarat, W, Petruşel, A, Kumam, P: Common coupled fixed point theorems for $w^{*}$-compatible mappings without mixed monotone property. Rend. Circ. Mat. Palermo 61, 361-382 (2012)

9. Sintunavarat, W, Kumam, P, Cho, YJ: Coupled fixed point theorems for nonlinear contractions without mixed monotone property. Fixed Point Theory Appl. 2012, 170 (2012)

10. Karapınar, E, Kumam, P, Sintunavarat, W: Coupled fixed point theorems in cone metric spaces with a c-distance and applications. Fixed Point Theory Appl. 2012, 194 (2012)

11. Abbas, M, Sintunavarat, W, Kumam, P: Coupled fixed point of generalized contractive mappings on partially ordered G-metric spaces. Fixed Point Theory Appl. 2012, 31 (2012)

12. Aydi, H, Damjanović, B, Samet, B, Shatanawi, W: Coupled fixed point theorems for nonlinear contractions in partially ordered G-metric spaces. Math. Comput. Model. 54, 2443-2450 (2011)

13. Aydi, H, Postolache, M, Shatanawi, W: Coupled fixed point results for $(\psi, \varphi)$-weakly contractive mappings in ordered G-metric spaces. Comput. Math. Appl. 63, 298-309 (2012

14. Cho, YJ, Rhoades, BE, Saadati, R, Samet, B, Shatanawi, W: Nonlinear coupled fixed point theorems in ordered generalized metric spaces with integral type. Fixed Point Theory Appl. 2012, 8 (2012)

15. Choudhury, BS, Maity, P: Coupled fixed point results in generalized metric spaces. Math. Comput. Model. 54, 73-79 (2011) 
16. Karapinar, E, Kaymakcalan, B, Taş, K: On coupled fixed point theorems on partially ordered G-metric spaces. J. Inequal. Appl. 2012, 200 (2012)

17. Karapinar, E, Kumam, P, Erhan, IM: Coupled fixed points on partially ordered G-metric spaces. Fixed Point Theory Appl. $2012,174(2012)$

18. Luong, NV, Thuan, NX: Coupled fixed point theorems in partially ordered G-metric space. Math. Comput. Model. 55, 1601-1609 (2012)

19. Mohiuddine, SM, Alotaibi, A: On coupled fixed point theorems for nonlinear contractions in partially ordered G-metric spaces. Abstr. Appl. Anal. 2012, Article ID 897198 (2012)

20. Nashine, HK: Coupled common fixed point results in ordered G-metric spaces. J. Nonlinear Sci. Appl. 1, 1-13 (2012)

21. Shatanawi, W: Coupled fixed point theorems in generalized metric spaces. Hacet. J. Math. Stat. 40, $441-447$ (2011)

22. Shatanawi, W, Abbas, M, Nazir, T: Common coupled coincidence and coupled fixed point results in two generalized metric space. Fixed Point Theory Appl. 2011, 80 (2011)

23. Wangkeeree, R: Coupled fixed point theorems for generalized contractive mappings in partially ordered G-metric spaces. Fixed Point Theory Appl. 2012, 172 (2012)

24. Jleli, M, Samet, B: Remarks on G-metric spaces and fixed point theorems. Fixed Point Theory Appl. 2012,210 (2012)

25. Samet, B, Vetro, C, Vetro, F: Remarks on G-metric spaces. Int. J. Anal. 2013, Article ID 917158 (2013)

26. Amini-Harandi, A: Coupled and tripled fixed point theory in partially ordered metric spaces with application to initial value problem. Math. Comput. Model. 57, 2343-2348 (2013)

27. Berinde, $\mathrm{V}$ : Generalized coupled fixed point theorems for mixed monotone mappings in partially ordered metric spaces. Nonlinear Anal. 74, 7347-7355 (2011)

28. Berinde, $\mathrm{V}$ : Coupled fixed point theorems for $\phi$-contractive mixed monotone mappings in partially ordered metric spaces. Nonlinear Anal. 75, 3218-3228 (2012)

29. Berinde, V: Coupled coincidence point theorems for mixed monotone nonlinear operators. Comput. Math. Appl. 64, 1770-1777 (2012)

30. Golubović, Z, Kadelburg, Z, Radenović, S: Coupled coincidence points of mappings in ordered partial metric spaces. Abstr. Appl. Anal. 2012, Article ID 192581 (2012)

31. Kadelburg, Z, Radenović, S: Coupled fixed point results under tvs-cone metric and $w$-cone-distance. Adv. Fixed Point Theory 2, 29-46 (2012)

32. Jleli, M, Rajić, VĆ, Samet, B, Vetro, C: Fixed point theorems on ordered metric spaces and applications to nonlinear elastic beam equations. J. Fixed Point Theory Appl. 12, 175-192 (2012)

33. Kadelburg, Z, Nashine, HK, Radenović, S: Coupled fixed point results in 0-complete ordered partial metric spaces. J. Adv. Math. Stud. 6, 159-172 (2013)

34. Kadelburg, Z, Nashine, HK, Radenović, S: Common coupled fixed point results in partially ordered G-metric spaces. Bull. Math. Anal. Appl. 4, 51-63 (2012)

35. Agarwal, RP, Karapinar, E: Remarks on some coupled fixed point theorems in G-metric spaces. Fixed Point Theory Appl. 2013, 2 (2013)

36. Abbas, M, llić, D, Khan, MA: Coupled coincidence point and coupled common fixed point theorems in partially ordered metric spaces with $w$-distance. Fixed Point Theory Appl. 2010, Article ID 134897 (2010)

37. Ćirić, LB, Cakić, N, Rajović, M, Ume, JS: Monotone generalized nonlinear contractions in partially ordered metric spaces. Fixed Point Theory Appl. 2008, Article ID 131294 (2008)

38. Nashine, HK, Kadelburg, Z, Radenović, S: Coincidence and fixed point results under generalized weakly contractive condition in partially ordered G-metric spaces. Filomat (to appear)

39. Ćirić, LB: A generalization of Banach's contraction principle. Proc. Am. Math. Soc. 45, 267-273 (1974)

40. Jachymski, J: Equivalent conditions for generalized contractions on (ordered) metric spaces. Nonlinear Anal. 74 , 768-774 (2011)

41. Boyd, DW, Wong, JS: On nonlinear contractions. Proc. Am. Math. Soc. 20, 458-464 (1969)

10.1186/1029-242X-2013-528

Cite this article as: Agarwal et al.: On coupled fixed point results in asymmetric G-metric spaces. Journal of Inequalities and Applications 2013, 2013:528

\section{Submit your manuscript to a SpringerOpen ${ }^{\circ}$ journal and benefit from:}

- Convenient online submission

- Rigorous peer review

- Immediate publication on acceptance

- Open access: articles freely available online

- High visibility within the field

- Retaining the copyright to your article

Submit your next manuscript at $>$ springeropen.com 